\title{
Value of Diffusion-Weighted and Perfusion-Weighted MR Imaging in Differentiation of Recurrent Tongue Carcinoma from Post-Treatment Changes
}

\author{
FATMA MOHAMED SHERIF, M.D.* and MAIY ABDEL-RAHEIM ALSHAHAT, M.D.** \\ The Departments of Radiology* and Clinical Oncology \& Nuclear Medicine**, Faculty of Medicine, Mansoura University
}

\begin{abstract}
Background: Tongue carcinoma is characterized by high recurrence rate. Thus, post-treatment follow-up imaging is critically important. Advanced Magnetic Resonance Imaging (MRI) techniques can be employed for this purpose due to the limited accuracy of conventional MRI.
\end{abstract}

Aim of Study: Evaluation of the value of Diffusion Weighted (DW) and perfusion weighted MRI implementation in the post-treatment follow-up of tongue carcinoma.

Patients and Methods: This study was conducted on 23 patients on post-treatment follow-up of tongue carcinoma. They underwent DW-MRI and Dynamic Contrast Enhanced (DCE) perfusion T1-weighted MRI to differentiate between recurrent tumor and post-treatment changes. Apparent Diffusion Coefficient (ADC) was estimated, perfusion MR qualitative and semiquantitative assessment was performed. Resulting data were compared to histopathologic characterization $(n=18)$ and further clinical and radiological follow-up $(n=5)$ which were considered as the reference standards.

Results: The mean $\mathrm{ADC}$ value of recurrent tongue carcinoma $(1.029 \pm 0.207 \times 10 \mathrm{~mm} / \mathrm{s})$ was significantly lower $(p<0.001)$ than the mean $\mathrm{ADC}$ value of post-treatment changes $\left(1.425 \pm 0.238 \times 10^{-3} \mathrm{~mm} / \mathrm{s}\right)$. The ADC threshold used for differentiating reçurrent tumor from post-treatment changes was $1.175 \times 10^{-3} \mathrm{~mm} / \mathrm{s}$ with Area Under the Curve (AUC) of 0.938 and diagnostic accuracy of $82.6 \%$. Among the DCE perfusion weighted MR parameters, the wash in rate and Area Under Gadolinium Curve (AUGC) displayed the highest diagnostic accuracy $(73.9 \%)$ with thresholds of $18.65 \mathrm{a} . \mathrm{u} / \mathrm{s}$ and $126826.7 \mathrm{~m}$.M.s respectively. The combined use of both DW-MRI and perfusion weighted MRI showed the highest diagnostic accuracy.

Conclusion: The DW MRI is a non-invasive technique providing accurate post-treatment follow-up assessment of tongue carcinoma. The perfusion weighted MRI provides lower diagnostic accuracy than DWI. The combined use of both techniques provides superior differentiation of tumor recurrence from post-treatment changes.

Correspondence to: Dr. Fatma Mohamed Sherif, E-Mail: fatema sherif@yahoo.com
Key Words: Tongue carcinoma-Diffusion-weighted MR imaging - Perfusion-weighted MR imaging Recurrence-Post-treatment changes.

\section{Introduction}

TONGUE carcinoma is the most common malignancy of the oral cavity, it represents about 30$40 \%$ of the oral cavity cancers $[1,2]$. The management of tongue carcinoma usually involves multidisciplinary treatment including surgery, radiation and chemotherapy [2-5]. Despite the constant advancement of surgery and radiation therapy, the treatment of tongue carcinoma is difficult due to the localization and the invasive nature of the available treatment methods, thus, post-treatment follow-up is crucial $[\mathbf{3 , 6}$.

Many cross-sectional imaging modalities are used for post-treatment follow-up of the tongue

\begin{tabular}{ll}
\hline List of Abbreviations: \\
MRI & : Magnetic Resonance Imaging. \\
DW-MRI & : Diffusion Weighted Magnetic Resonance \\
& Imaging. \\
DCE MRI & : Dynamic Contrast Enhanced Magnetic \\
& Resonance Imaging. \\
ADC & : Apparent Diffusion Coefficient. \\
AUC & : Area Under Curve. \\
AUGC & : Area Under Gadolinium Curve. \\
a.u/s & : Arbitrary unit/second. \\
m.M.s & : Millimole. Second. \\
CT & $:$ Computed Tomography. \\
18-FFDG-PET & $:$ 18- F-Fluorodeoxyglucose Positron Emission \\
& Tomography. \\
TR/TE/NEX & $:$ Repetition time/echo time/number of \\
& excitation. \\
FOV & $:$ Field of View. \\
TIC & $:$ Time signal intensity curve. \\
TTP & $:$ Time-to-Peak. \\
ROC & $:$ Receiver Operator Characteristic. \\
PPV & $:$ Positive Predictive Value. \\
NPP & : Negative Predictive Value. \\
DSC & $:$ Dynamic Susceptibility Contrast.
\end{tabular}


cancers, such as Computed Tomography (CT), Magnetic Resonance Imaging (MRI) and 18-FFluorodeoxyglucose Positron Emission Tomography (18-FFDG-PET) [3,7]. MRI is regarded the preferred imaging modality in diagnosis of oral area tumor detection and follow-up as well. However, surgery and radiation can cause significant inflammation, fibrosis and distortion of the anatomy preventing accurate differentiation between residual or recurrent tumor and post-treatment changes by conventional MRI sequences [1,2,7-10].

New functional imaging technologies nowadays are employed in oncologic post-treatment followup due to the limited accuracy of conventional MRI. Diffusion Weighted Imaging (DWI) is an MR technique that has been suggested as noninvasive imaging biomarker to predict tumor response. The obvious advantage of DW-MRI, which uses water molecules motion in intracellular and extracellular space to reflect biological changes in tumor microenvironment, is that it is useful for accurately distinguishing recurrent disease from radiationinduced soft-tissue changes. The DW-MRI derived parameter of Apparent Diffusion Coefficient (ADC) represents direction independent water displacement. In tissues with high cellularity, free water motion is restricted, and the measured ADC is low, whereas in tissues with low cellularity, the corresponding ADC is high. This inverse correlation between ADC and cellularity may serve as a method to differentiate between recurrent tumors and posttreatment changes. DW-MRI should detect the disease before the recurrence of clinical symptoms $[3,5,7,11-13]$

Another advanced MRI technique that can be useful in the same purpose is perfusion-weighted Dynamic Contrast-Enhanced (DCE) MRI using T1-weighted sequence. This technique has been reported to be able to characterize perfusion and vascularization of tissues. Therefore, it can play a key role as a beneficial biomarker for tumor angiogenesis. It has also been demonstrated that DCE MRI can be helpful in differentiation between malignant and benign lesions as well as prediction of response to therapy in different tumors. It involves serial MR image acquisition after injection of gadolinium-based contrast agent, thus, tissue perfusion and permeability can be assessed based on the signal enhancement kinetics [6,13-19]

This study aimed at assessment of the value of DW-MRI and perfusion-weighted DCE MRI as well as their combined use in the post-treatment follow-up of tongue carcinoma.

\section{Patients and Methods}

Study design and patients:

The Institutional review board approved this longitudinal study and informed consents were obtained from participating patients. From July 2018 to January 2020 , this study was conducted in Mansoura University Hospital, Radiology Department upon 23 consecutive patients (14 males, 9 females) with their mean age of 52.5 years, ranging from 38 to 63 years. All patients were referred from Clinical Oncology Department, they were on follow-up after ending the treatment of pathologically confirmed tongue carcinoma. All cases had been treated surgically with postoperative radiation therapy.

\section{Inclusion criteria:}

Patients with treated histopathologically-proven tongue squamous cell carcinoma, the term between the end of treatment and post-treatment imaging was 6 months or longer.

\section{Exclusion criteria:}

Patients with tongue carcinoma who were still having radiotherapy or had less than 6 months duration since the end of their treatment, contraindications to gadolinium contrast administration such as renal impairment or known allergic reaction to gadolinium, contraindication to MR examination like having artificial pacemakers and uncooperative patients.

The final diagnosis was obtained with histopathologic characterization $(\mathrm{n}=18)$ and further clinical and radiological follow-up $(n=5)$ which were considered the reference standards in this study.

\section{MR image acquisition:}

All patients underwent MR studies using a 1.5Tesla scanner (Ingenia, Philips, Philips Medical Systems, Netherland). Dedicated multichannel head and neck coil was applied. Non-contrast MRI examination included the following sequences: Axial T1 weighted Turbo Spin Echo (TSE) without and with fat suppression with the following parameters: Repetition time/echo time/number of excitation $(\mathrm{TR} / \mathrm{TE} / \mathrm{NEX})=(600 / 20 / 2)$, axial T2 TSE without fat suppression and coronal T2 weighted sequence with fat suppression with the following parameters: $(\mathrm{TR} / \mathrm{TE} / \mathrm{NEX})=(4000 / 90 / 4)$; field of view $(\mathrm{FOV})=18 \mathrm{~cm}$; section thickness, $2 \mathrm{~mm}$; section gap, 1mm; matrix, $256 \times 256$.

The DW-MRI was carried out using multi-slice single shot spin echo planar imaging sequence in 
the axial plane with the following parameters: TR/ TE of 1700/100msec; FOV of $25 \mathrm{~cm}$; section thickness of $5 \mathrm{~mm}$; inter-slice gap of $2 \mathrm{~mm}$ and acquisition matrix of $256 \times 128$. Diffusion gradients were applied in the three orthogonal directions ( $\mathrm{x}, \mathrm{y}$ and z) with the same strength. DW-MR images were acquired with two diffusion weighted factors, factor $\mathrm{b}$ of 0 and $1000 \mathrm{~s} / \mathrm{mm}^{2}$ in the axial plane. The acquisition time for the diffusion-weighted sequence was 1 minute and 45 seconds. Apparent Diffusion Co-efficient (ADC) maps were generated for all images, an ADC maps were obtained.

Perfusion weighted MRI was obtained with dynamic 3D (axial contrast-enhanced T1WI with fat suppression) fast spoiled gradient recalled sequence was performed using the following parameters: TR/TE=5.1/1.1 msec; flip angle $30 \mathrm{de}-$ grees; FOV $18 \mathrm{~cm}$; section thickness $4 \mathrm{~mm}$; section gap $1 \mathrm{~mm}$; matrix $256 \times 128$, total acquisition time of 300 seconds during bolus injection of a single dose contrast agent (gadolinium-DTPA) at a concentration of $0.1 \mathrm{mmol} / \mathrm{kg}$ at a rate of $2.5 \mathrm{ml} / \mathrm{s}$ given intravenously, followed by $20 \mathrm{ml}$ saline flush using an automatic injector. Sequential images were obtained through the lesion in axial plane and at different time intervals (at 30, 60, 90, 120, 150, 180, 240 and 300s following injection).

After dynamic Contrast-Enhanced (CE) image acquisition, conventional CE T1-weighted fat suppressed MR sequence was acquired in the axial, sagittal, and coronal planes with the same parameters as non-contrast axial T1 weighted sequence.

\section{MR image analysis:}

The MR images were analyzed on a Philips extended work space release 2.6 workstation. Conventional MR images were analyzed for abnormal signal intensity lesions and contrast enhancement on contrast-enhanced T1 weighted images.

Qualitative assessment of DW-MRI study was performed, diffusion restriction was decided as high signal intensity lesions on high b-value (1000) diffusion weighted images with hypointense signal on corresponding ADC map. Quantitative analysis of ADC values of the lesions was performed on ADC map. Circular Region of Interest (ROI), measuring $10-15 \mathrm{~mm}$ in diameter, was placed in the lesion (avoiding cystic parts in mixed lesion by correlation with $\mathrm{T} 2$ and post contrast $\mathrm{T} 1$ weighted images) and three $\mathrm{ADC}_{2}$ values were automatically measured in $\mathrm{X}_{10} 0^{-3} \mathrm{~mm}^{2} / \mathrm{sec}$, then their mean ADC value was calculated.
Dynamic contrast enhanced images were assessed qualitatively and semiquantitatively. Region of Interest (ROI) was plaçed within an area of the tumor measuring $10 \mathrm{~mm}^{2}$ (avoiding cystic parts, vessels, necrosis, calcifications, and hemorrhages). Qualitative assessment was concerned with visual assessment of time signal intensity curve (TIC), it was referred to as type I (persistent), type II (plateau) or type III (washout) curves. Semi-quantitative parameters: Wash-in rate, wash-out rate, Area Under Gadolinium Curve (AUGC) and Time-topeak (TTP) were automatically calculated.

\section{Statistical analysis:}

Data were analysed using Statistical Package for Social Science version 22 (IBM SPSS Inc. released 2013, Chicago, Ill, USA). Qualitative data were described using number and percent. Quantitative data were declared using median (minimum and maximum) for non-parametric data and mean, standard deviation for parametric data. Significance of the obtained results was judged at $p \leq .05$. Fischer Exact test was used for comparison of qualitative data (diffusion restriction, type of DCE TIC) in comparison to the reference standard results. Parametric and non parametric data were compared using Student $t$-test \& Mann-Whitney U-test respectively. Receiver Operator Characteristic (ROC) curve analysis was performed to detect validity of ADC \& perfusion parameters in differentiating recurrent tongue carcinoma from post-treatment changes. Sensitivity and specificity were detected from the curve, Positive Predictive Value (PPV), Negative Predictive Value (NPV) and accuracy were calculated through cross tabulation.

\section{Results}

This study comprised 23 patients on follow-up 6-24 months after completion of treatment of tongue carcinoma, 14 males and 9 females, their mean age was $52.52 \pm 7.71 \mathrm{SD}$. The treated tumors had been pathologically diagnosed as squamous cell carcinoma and located in the oral tongue $(n=16)$ and at the tongue base $(n=7)$.

The final diagnosis was acquired with histopathologic characterization $(\mathrm{n}=18)$ and further clinical and radiological follow-up $(n=5)$. The final diagnosis was tumor recurrence in 13 patients and post-treatment changes in 10 patients. The time of tumor recurrence was 6-12 months after completion of therapy $(n=4), 12-18$ months after completion of therapy $(n=7), 18-24$ months after completion of therapy $(n=2)$. 
Restricted diffusion was detected in $11(84.6 \%)$ of recurrent tumor cases, non restricted diffusion was predicted in $90.0 \%$ of post-treatment change cases. The mean ADC value for tumor recurrence was $1.029 \pm 0.207 \times 10^{-3} \mathrm{~mm} / \mathrm{s}$ Fig. (1) and for post-treatment changes was $1.425 \pm 0.238 \times 10$ $\mathrm{mm}^{2} / \mathrm{s}$ Fig. (2) with statistically significant difference between them $(p<0.001)$ (Table 1). The Receiver-Operator Characteristics (ROC) analysis declared that the threshold of ADCS used to differentiate between tumor recurrençe and posttreatment changes was $1.175 \times 10^{-5} \mathrm{~mm} / \mathrm{s}$. With diagnostic accuracy of $82.6 \%$, sensitivity of $84.6 \%$ and specificity of $80 \%$ (Table 2) Fig. (3).

Qualitative assessment of DCE MR study revealed washout (type III) curve in $69.2 \%$ of tumor recurrence cases Fig. (1), while plateau curve was depicted in $60 \%$ of cases of post-treatment changes Fig. (2). Regarding quantitative DCE MR parameters, no detected significant difference of between tumor recurrence and post-treatment changes (Table 1). The ROC analysis revealed that among the DCE MR parameters, the wash in and wash out rates showed the highest sensitivity to differentiate between tumor recurrence and post-treatment changes $(69.2 \%$ for each), the thresholds of wash in rate and washout rates for differentiation between both entities were $18.65 \mathrm{a} . \mathrm{u} / \mathrm{s}$ and $2.25 \mathrm{a}$.u/s respectively. Meanwhile, the AUGC displayed the highest specificity for differentiation between tumor recurrence and post-treatment changes (90\%) with a threshold of $126826.7 \mathrm{~m}$.M.s for differentiation of both entities (Table 2) Fig. (4).

The ROC analysis of results of combined implementation of DWI and DCE MR for differentiation of tongue carcinoma post-treatment recurrence and post-treatment changes revealed the highest sensitivity, specificity and accuracy (Table 2), Fig. (5).

Table (1): ADC value \& perfusion MR parameters in recurrent tongue carcinoma from post-treatment changes.

\begin{tabular}{|c|c|c|c|}
\hline & $\begin{array}{l}\text { Post radiation change } \\
\qquad \mathrm{N}=10\end{array}$ & $\begin{array}{l}\text { Recurrent tumor } \\
\qquad \mathrm{N}=13\end{array}$ & $\begin{array}{c}\text { Test of } \\
\text { significance }\end{array}$ \\
\hline \multicolumn{4}{|l|}{ DWI $n(\%):$} \\
\hline No restriction & $9(90.0 \%)$ & $2(15.4 \%)$ & FET \\
\hline Restriction & $1(10.0 \%)$ & $11(84.6 \%)$ & $p<0.001 *$ \\
\hline \multicolumn{4}{|l|}{$A D C\left(\times 10^{-3} \mathrm{~mm}^{2} / \mathrm{s}\right):$} \\
\hline Mean \pm SD & $1.425 \pm 0.238$ & $1.029 \pm 0.207$ & $t=4.249, p<0.001 *$ \\
\hline \multicolumn{4}{|l|}{ Wash in $(a . u / s)$ : } \\
\hline Median (range) & $17.1(14.0-20.6)$ & $21.0(12.2-78.9)$ & $\mathrm{Z}=1.83, p=0.067$ \\
\hline \multicolumn{4}{|l|}{ Wash out $(a . u / s)$ : } \\
\hline Median (range) & $1.9(0.12-13.8)$ & $3.8(0.0-20.2)$ & $\mathrm{Z}=0.528, p=0.598$ \\
\hline \multicolumn{4}{|l|}{$A U G C(m . M . s):$} \\
\hline Median (range) & $94358.0(33100-132000)$ & $140590(17800-203000)$ & $Z=1.426, p=0.154$ \\
\hline \multicolumn{2}{|l|}{ Time to peak $(s)$ : } & $118.0(51.6-231.8)$ & $\mathrm{Z}=2.14, p=0.094$ \\
\hline \multicolumn{4}{|l|}{ Types of curve: $n(\%)$ : } \\
\hline Type II (Plateau) & $6(60.0)$ & $4(30.8)$ & FET \\
\hline Type III (Washout) & $4(40.0)$ & $9(69.2)$ & $p=0.222$ \\
\hline $\begin{array}{l}\text { FET : Fischer Exact Test. } \\
Z \text { : Mann Whitney U-te }\end{array}$ & $\begin{array}{l}t \quad: \text { Studen } \\
\text { a.u }: \text { Arbitra }\end{array}$ & & $\begin{array}{l}\text { llimole. } \\
\text { tistically significant. }\end{array}$ \\
\hline
\end{tabular}

Table (2): Validity of ADC value, perfusion MR parameters and their combined implementation in differentiating recurrent tongue carcinoma from post-treatment changes.

\begin{tabular}{lllccccc}
\hline & AUC (95\% CI) & \multicolumn{1}{c}{$\begin{array}{c}\text { Cut off } \\
\text { point }\end{array}$} & $\begin{array}{c}\text { Sensitivity } \\
(\%)\end{array}$ & $\begin{array}{c}\text { Specificity } \\
(\%)\end{array}$ & $\begin{array}{c}\text { PPV } \\
(\%)\end{array}$ & $\begin{array}{c}\text { NPV } \\
(\%)\end{array}$ & $\begin{array}{c}\text { Accuracy } \\
(\%)\end{array}$ \\
\hline - ADC (X 10 $\left.\mathrm{mm}^{-3} / \mathrm{s}\right)$ & $0.938(0.842-1.0)$ & $\leq 1.175$ & 84.6 & 80.0 & 84.6 & 80.0 & 82.6 \\
- Wash in (a.u/s) & $0.727(0.505-0.949)$ & $\geq 18.65$ & 69.2 & 80.0 & 66.7 & 81.8 & 73.9 \\
- Wash out (a.u/s) & $0.565(0.322-0.809)$ & $\geq 2.25$ & 69.2 & 60.0 & 69.2 & 60.0 & 65.2 \\
- AUGC (m.M.s) & $0.677(0.438-0.915)$ & $\geq 126826.7$ & 61.5 & 90.0 & 88.9 & 64.3 & 73.9 \\
- Time to peak (s) & $0.708(0.491-0.925)$ & $\geq 111.5$ & 61.5 & 50.0 & 61.5 & 50.0 & 56.5 \\
- Combined ADC \& perfusion & $1.0(1.0-1.0)$ & 0.99 & 100.0 & 100.0 & 100.0 & 100.0 & 100.0 \\
MRI parameters & & & & & & & \\
\hline
\end{tabular}

AUC : Area Under Curve.

$\mathrm{CI}$ : Confidence Interval.
PPV : Positive Predictive Value. NPV : Negative Predictive Value. 

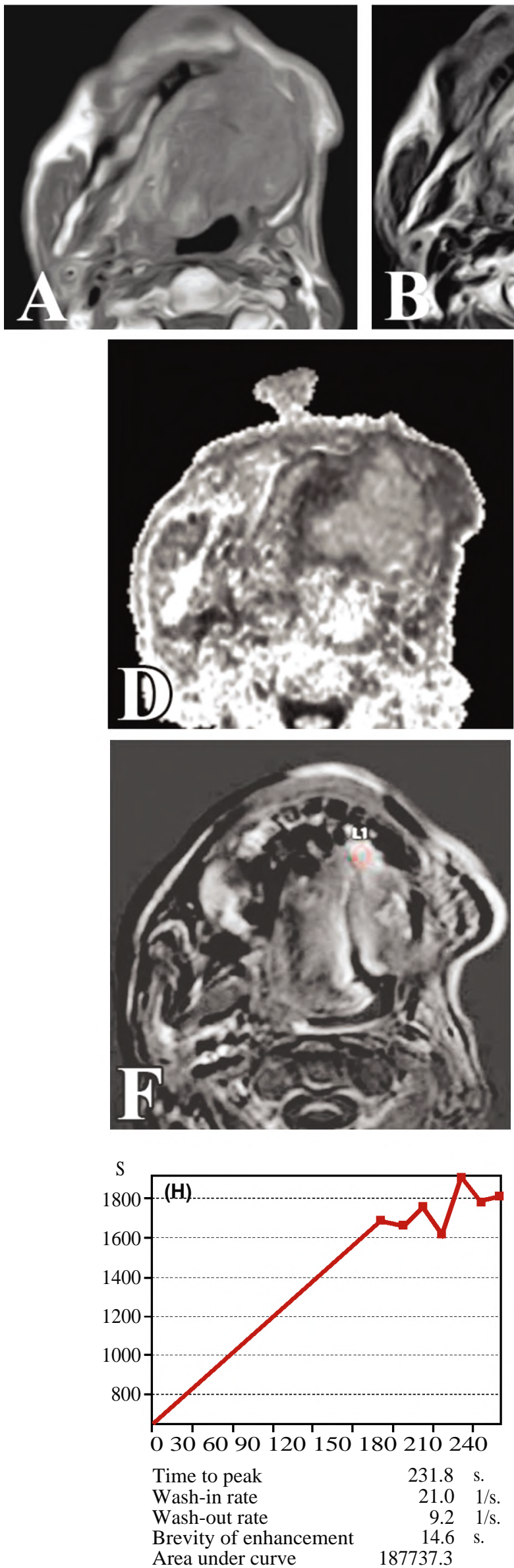
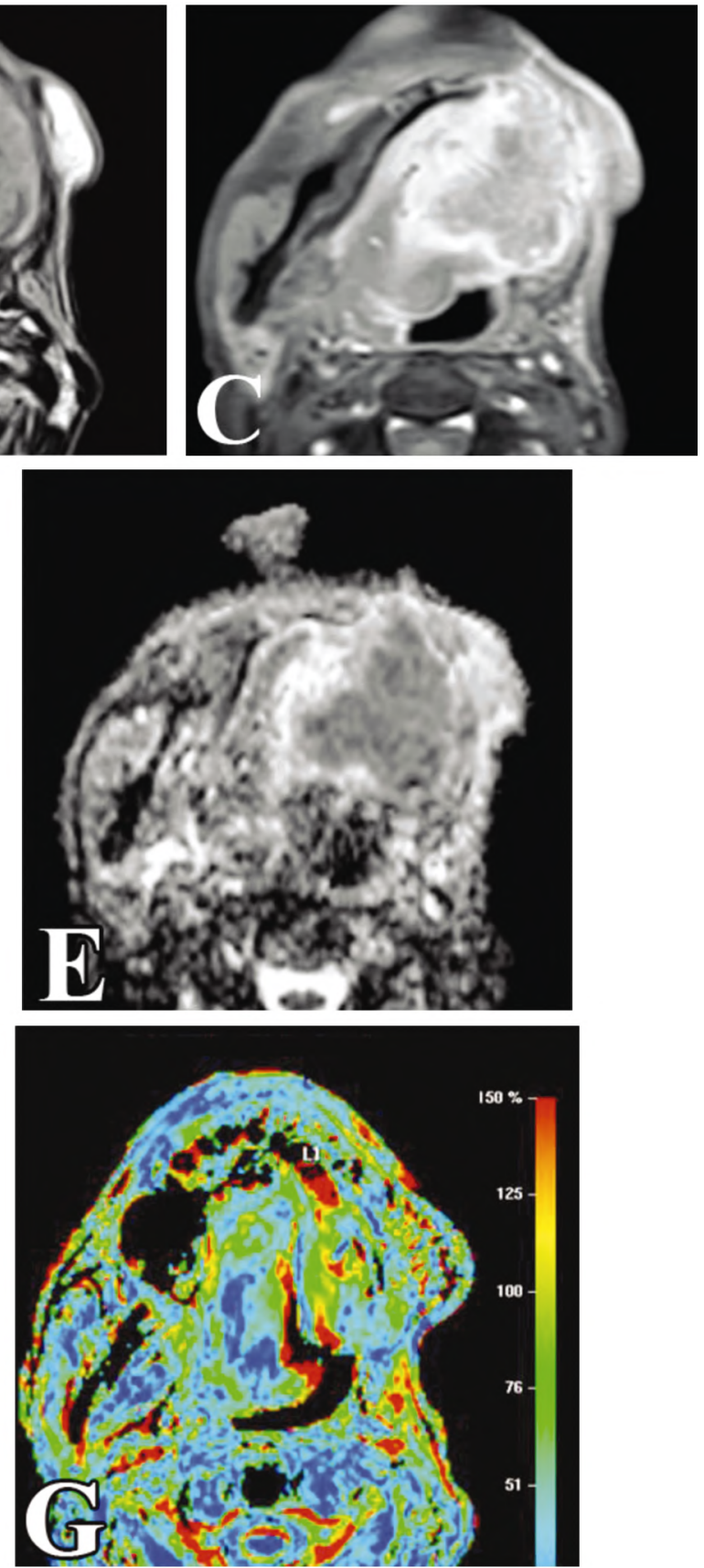

Fig. (1): MRI of a 44 years old male on posttreatment follow-up of tongue squamous cell carcinoma after 24 months termination of postoperative radiation therapy, with recurrent tumor (Pathologically proved): Axial T1 (A), axial T2 (B) showed abnormal signal intensity soft tissue mass at the surgical bed on the left side of tongue, with heterogeneous enhancement on contrastenhanced axial T1 (C) and area of diffusion restriction DWI-MRI (D) and ADC map (E), with mild enhancement on DCE MR subtraction image (F) (arrow). DCE MR perfusion colored map (G), time intensity curve (TIC) (H) displayed type III (washout) curve. 

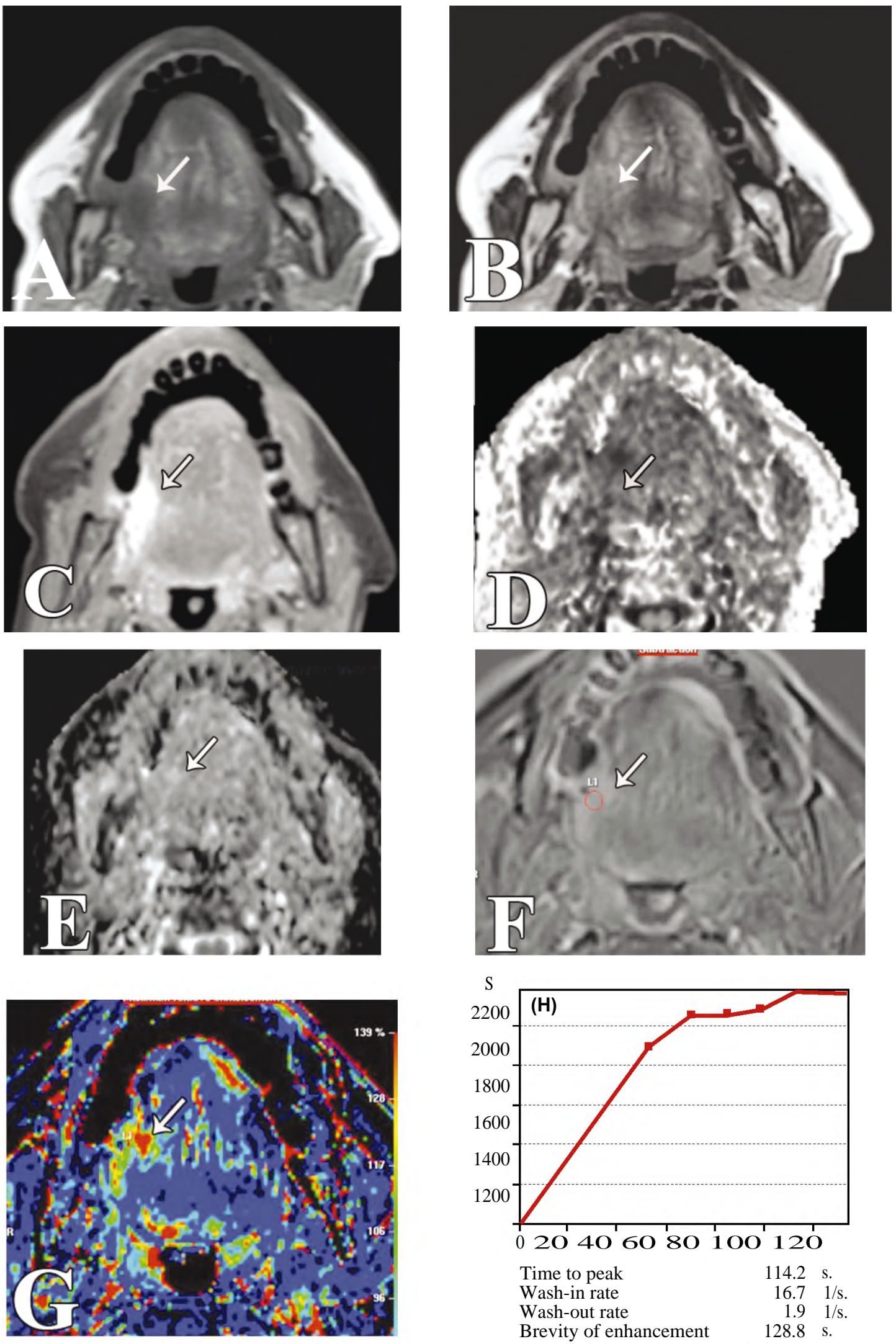

Fig. (2): MRI of a 59 year old female on post-treatment follow-up of tongue squamous cell carcinoma after 10 months termination of postoperative radiation therapy, with post-treatment changes (confirmed by further follow-up): Axial T1 (A), axial T2 (B) show abnormal signal intensity lesion at the surgical bed on the right side of tongue, enhancing on contrast-enhanced axial T 1 (C), False diffusion restriction on DWI-MRI (D) and no diffusion restriction on ADC map (E), with mild enhancement on DCE MR subtraction image (F) (arrow). DCE MR perfusion colored map (G), time intensity curve (TIC) (H) shows type II (plateau) curve. 


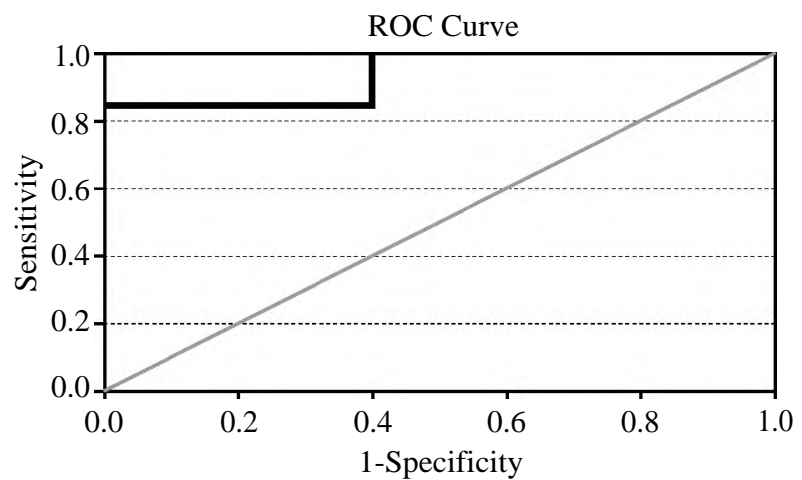

Fig. (3): Receiver Operating Characteristics (ROC) of ADC value in differentiating recurrent tongue carcinoma from post-treatment changes.

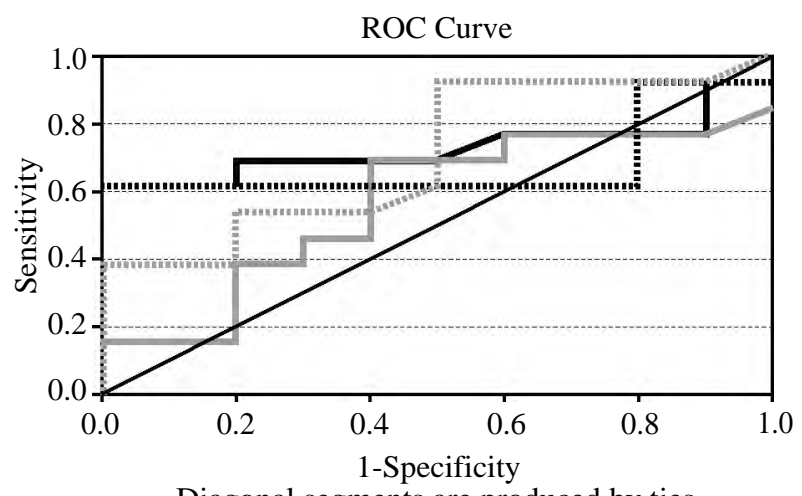

Diagonal segments are produced by ties

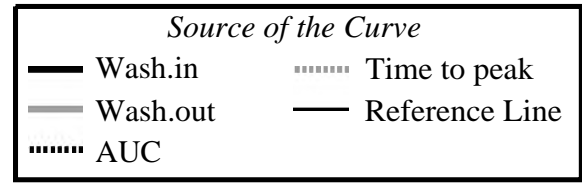

Fig. (4): Receiver Operating Characteristics (ROC) of perfusion MR parameters in differentiating recurrent tongue carcinoma from post-treatment changes.

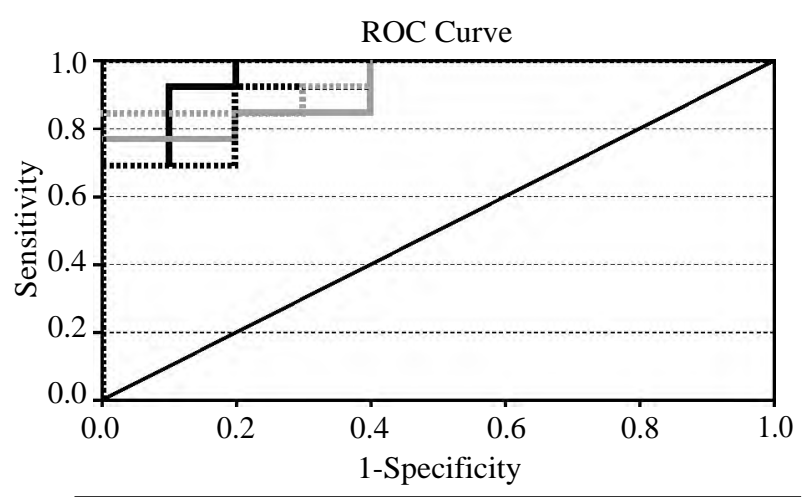

Source of the Curve

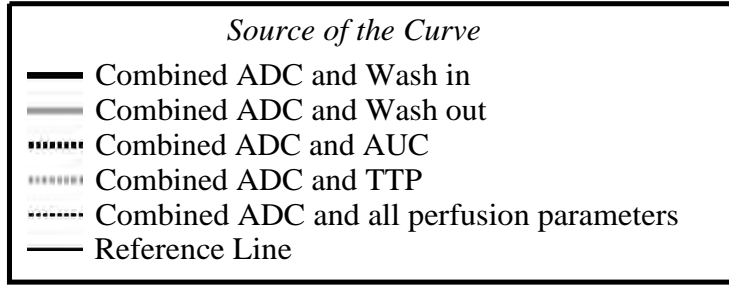

Fig. (5): Receiver Operating Characteristics (ROC) of combined ADC value \& perfusion MR parameters in differentiating recurrent tongue carcinoma from posttreatment changes.

\section{Discussion}

The present study investigated the value of diffusion weighted MRI and perfusion weighted MRI using DCE T1-weighted MRI in posttreatment evaluation of tongue carcinoma. The quantitative diffusion weighted MRI reported high sensitivity, specificity and accuracy of ADC study in differentiation between post-treatment changes and tumor recurrence. Among the perfusion parameters, the wash in and wash out rates showed moderate sensitivity and the AUGC displayed the highest specificity to differentiate between tumor recurrence and post-treatment changes. However, combined use of both diffusion and perfusion MR techniques revealed excellent sensitivity, specificity and accuracy.

Due to the high recurrence rate of tongue carcinoma, post-treatment surveillance is critically important. Magnetic resonance imaging is the most preferable technique in this issue. However, posttreatment morphological findings from conventional MRI has limited accuracy because of complexity of the surgeries performed and the post-irradiation changes. Therefore, new functional imaging technologies are being employed $[\mathbf{3 , 5 , 7 , 2 0 ]}$.

Diffusion-weighted MRI and its related ADC have been reported to be useful tool to differentiate tumor recurrence from normal post-treatment changes, it has been considered one of the promising recently developed techniques for functional assessment of tumors as well as characterization of regional lymph nodes if present, detection of cancer recurrence and, more recently, assessment of treatment response in different organs or tissues. Nevertheless, its use is still limited in the clinical practice of head and neck oncology $[\mathbf{5 , 1 1 , 1 3 , 2 1 , 2 2 ]}$.

In the current study, DW-MRI study revealed that the ADC value of recurrent tongue carcinomas was significantly lower than that of post-treatment changes $(p<0.001)$. The ADC cutoff ${ }_{-3}$ value between $_{2}$ the two groups was $1.175 \times 10^{-3} \mathrm{~mm}^{2} / \mathrm{s}$. With accuracy of $82.6 \%$. These results matched with previous studies conducted on role of DW-MRI in post-treatment follow-up of squamous cell carcinoma in the head and neck region [21,23-25].

In a study concerned with patients laryngeal and hypopharyngeal malignancy treated with chemo/radiotherapy, the mean ADC value of residual/recurrent tumors were significantly lower than that of post-radiation changes $(p<0.0002)$ and ROC analysis provided an ADC threshold measuring $1.3 \times 10 \mathrm{~mm} / \mathrm{s}$ with sensitivity of $67 \%$, specif- 
icity of $86 \%$ and accuracy of $78 \%$ [26]. Ailianou et al analyzed 100 cases of treated head and neck squamous cell carcinoma, matching to our results, they reported a threshold ADC value of $1.22 \mathrm{X}$ $10 \mathrm{~mm} / \mathrm{s}$ for differentiation between recurrent malignancy and post-treatment changes with sensitivity of $78.9 \%$ and specificity of $86.1 \%$ [27]

Perfusion weighted MRI permits the detection of the contrast kinetics within the tumors. The perfusion weighted MRI methods include T1weighted DCE MRI and T2*-weighted Dynamic Susceptibility Contrast (DSC) perfusion weighted MRI which uses echo-planar sequence. The T1weighted DCE MRI has higher spatial resolution than the T2*-weighted DSC MRI which may be associated with susceptibility artifact at the air/ tissue interface $[\mathbf{1 9 , 2 8}]$. The DCE MRI has been reported to be useful for various applications in head and neck oncology, such as differentiating squamous cell carcinoma from lymphoma, detecting metastatic lymph nodes, assessing tumor cell proliferation, predicting early treatment response and treatment outcome $[14,15,19,29,30]$

In the present study, qualitative assessment of DCE MRI revealed type III (washout curve) in $69.2 \%$ of recurrent tumor cases, while type II (plateau curve) in $60 \%$ of post-treatment changes cases. This result was explained that the small amount of interstitium in between the malignant cells may result in decreased leakage from blood vessels. While, the post-treatment inflammatory and fibrotic changes result in a large capacity of the interstitium which may drive contrast medium leakage from the blood vessels [28,31].

In the semi-quantitative assessment of this study, the wash in and wash out rates of recurrent tumor were higher than those of post-treatment changes, among the measured perfusion MR parameters, the wash in and wash out rates showed the highest sensitivity. These results were attributed to the nature of malignant tissue which was reported to by showing early enhancement and early washout out of gadolinium contrast [28]. In agreement with previous studies, the AUGC of recurrent malignancy was reported to be more than of the posttreatment changes, with the highest specificity among the measured DCE parameters. The AUGC reflects the accumulation and/or depletion of contrast media within the cancer regardless of curve shape, but it has no direct correlation with the contrast circulation pathway and it is not able to reflect any specific physiological processes such as perfusion or permeability $[\mathbf{2 8 , 3 2 ]}$. The recurrent malignant tumors displayed longer TTP than those of post-treatment changes, agreeing with Furukawa et al., who stated that benign lesions had faster TTP and washout times, indicating rather intact microvascular circulation with limited permeability. Also, this is likely due to leakiness of blood vessels in malignant tumors, thus taking longer time to reach the maximum [28]. Nevertheless, it was noticed in the present study that TTP parameter revealed the least sensitivity and specificity among the calculated perfusion parameters. The addition of DW-MRI to DCE MRI increased the confidence of prediction of differentiation of recurrent tongue carcinoma from post-treatment changes.

Our study had many advantages, including the combined study of two advanced MR techniques and their role in follow-up of tongue carcinoma, also the choice of the long term follow-up of cases after the resolution of early post-treatment reactions. However, there were some drawbacks in this study, including the small number of patients, the heterogeneity of the DCE MRI studies in terms of used sequences and reference standards, making it difficult to have reliable standardized quantitative results.

\section{Conclusion:}

Diffusion weighted MRI was declared to be a reliable fast non-invasive technique to differentiate recurrent tongue carcinoma from post-treatment changes. Perfusion weighted MRI showed less accuracy for this purpose. The combined use of both techniques increased the confidence of posttreatment follow-up of tongue carcinoma.

\section{References}

1- YANG X., ZHU J., DAI Y., TIAN Z., YANG G., SHI H., et al.: Multi-parametric effect in predicting tumor histological grade by using susceptibility weighted magnetic resonance imaging in tongue squamous cell carcinoma. BMC Medical Imaging, 19: 24, 2019.

2- ZU Y., NARAYANAN S., KIM Y., NAYAK K., BRONSON-LOWE K., VILLEGAS B., et al.: Evaluation of swallow function after tongue cancer treatment using real time magnetic resonance imaging: A pilot study. JAMA Otolaryngol. Head Neck Surg., 139 (12): 1312-9, 2013.

3- SIATO N., NADGIR R., NAKAHIRA M., TAKAHASHI M., UCHINO A., KIMURA F., et al.: Post-treatment CT and MR imaging in head and neck cancer: What the radiologist needs to know. RadioGraphics, 32: 1261-82, 2012.

4- ONG C. and CHONG V.: Imaging of tongue carcinoma. Cancer Imaging, 6: 186-93, 2006.

5- MARTINS E., CHOJNIAK R., KOWALSKI L., NICOLAU U., LIMA E. and BITENCOURT A.: Diffusion- 
Weighted MRI in the Assessment of Early Treatment Response in Patients with Squamous-Cell Carcinoma of the Head and Neck: Comparison with Morphological and PET/CT Findings. PLOS ONE, 10 (11): e0140009, 2015.

6- PATASZ P., ADAMSKI L., GÓRSKA-CHRZASTEK M., STARZYNSKA A. and STUDNIAREK M.: Contemporary diagnostic imaging of oral squamous cell carcinoma-A review of literature. Pol. J. Radiol., 82: 193-202, 2017.

7- De FELICE F., MUSIO D. and TOMBOLINI V.: Followup in head and neck cancer: A management dilemma. Advances in otolagrngology, http://dx.doi.org/10.1155/ 2015/703450, 2015.

8- TANTIWONGOKOSHI B., YU F., KANARD A. andMILLER F.: Role of 18F-FDG PET/CT in pre and posttreatment evaluation in head and neck carcinoma. World J. Radiol., 6 (5): 177-91, 2014.

9- MOSCI C., D.G. and QUAN A.: FDG-PET/CT Initial and Subsequent Therapy Evaluation: Progressing to PET/MR Imaging. PET Clin., 7: 369-80, 2012.

10- VAROQUAUX A., RAGER O., DULGUEROV P., BURKHARDT K., AILIANOU A. and BECKER M.: Diffusionweighted and PET/MR Imaging after Radiation Therapy for Malignant Head and Neck Tumors. RadioGraphics, 35 (5): 1502-27, 2015.

11- PAYABVASH S.: Quantitative diffusion magnetic resonance imaging in head and neck tumors. Quant Imaging Med. Surg., 8 (10): 1052-65, 2018.

12- GUO L., WANG G., FENG Y., YU T., GUO Y., BAI X. and YE Z.: Diffusion and perfusion magnetic resonance imaging for tumor volume definition in radiotherapy of brain tumors. Radiation Oncology, 11: 123-36, 2016.

13- WIDMANN G., HENNINGER B., KRESMER C. and JASCHKE W.: MRI sequences in head and neck radiologyState of the art. Fortschr. Röntgenstr., 189: 413-22, 2017.

14- CHEN L., YE Y., CHEN H., CHEN S., JIANG J., et al.: Dynamic contrast-enhanced magnetic resonance imaging for differentiating between primary tumor, metastatic node and normal tissue in head and neck cancer. Current Medical Imaging Reviews, 14: 416-21, 2018.

15- KIM S., LOEVNER L., QUON H., KILGER A., SHERMAN E., WEINSTEIN G., et al.: Prediction of response to chemoradiation therapy in squamous cell carcinomas of the head and neck using dynamic contrast-enhanced MR imaging. AJNR Am. J. Neuroradiol., 31: 262-8, 2010.

16- BERNSTEIN J., HOMER J. and WEST C.: Dynamic contrast-enhanced magnetic resonance imaging biomarkers in head and neck cancer: Potential to guide treatment? A systematic review. Oral Oncol., 50: 963-70, 2014.

17- SUROV A., MEYER H., GAWLITZA M., HOHN A., BOEHM A., KAHN T. and STUMPP P.: Correlation between DCE MRI and histopathological parameters in head and neck squamous cell carcinoma. Translational oncology, 10 (1): 17-21, 2017.

18-TEO Q., THNG C., KOH T. and NG Q.: Dynamic Contrastenhanced Magnetic Resonance Imaging: Applications in Oncology. Clinical Oncology, 26 (10): e9-e20, 2014.

19- GADDIKERI S., GADDIKERI R., TAILOR T. and ANZAI Y.: Dynamic contrast-enhanced MR imaging in head and neck cancer: Techniques and clinical applications. AJNR Am. J. Neuroradiol., 37: 588-95, 2016.

20- TROJANOWSKA A.: Squamous cell carcinoma of the head and neck-The role of diffusion and perfusion imaging in tumor recurrence and follow-up. Reports of Practical Oncology \& Radiotherapy, 16 (6): 207-12, 2011.

21- PILUDU F., GANGEMI E., MARUCCI L., GUERISSI A., PELLINI R., COVELLO R., et al.: MRI evaluation using DWI and T2WI of residual lymph nodes in patients affected by head and neck squamous cell carcinoma treated with chemo-radiotherapy. Current medical imaging, 14 (4): 599-608, 2018.

22- BABA A., OJIRI H., IKEDA K., YAMAUCHI H., OGINO N., SETO Y., et al.: Essentials on Oncological Imaging: Post-operative Computed Tomography and Magnetic Resonance Imaging of Oral Tongue Cancer. Canadian Association of Radiologists Journal, 69 (4): 458-67, 2018.

23- ABDEL RAZEK A., KANDEEL A.Y., SOLIMAN N. EL-SHENSHAWY H.M., KAMEL Y., NADA N. and DENEWAR A.: Role of Diffusion-Weighted Echo-Planar MR Imaging in Differentiation of Residual or Recurrent Head and Neck Tumors and Post-treatment Changes. American Journal of Neuroradiology, 28 (6): 1146-52, 2007.

24- ZHANG S., ZHOU S., SHANG D.S., BAO Y., RUAN L. and WU T.: The diagnostic role of diffusion-weighted magnetic resonance imaging in hypopharyngeal carcinoma. Oncology Letters, 15: 5533-44, 2018.

25- HWANG I., CHOI S.H., KIM Y.J., KIM K.G., LEE A.L., YUN T.J., KIM J.H. and SOHN C.H.: Differentiation of Recurrent Tumor and Post-treatment Changes in Head and Neck Squamous Cell Carcinoma: Application of High b-Value Diffusion-Weighted Imaging. American Journal of Neuroradiology, 34 (12): 2343-8, 2013.

26- TSHERING VOGEL D.W., ZBAEREN P., GERETSCHLAEGER A., VERMATHEN P., De KEYZER F. and THOENY H.C.: Diffusion-weighted MR imaging including bi-exponential fitting for the detection of recurrent or residual tumour after (chemo) radiotherapy for laryngeal and hypopharyngeal cancers. European Radiology, 23 (2): 562-9, 2012.

27- AILIANOU A., MUNDADA P., De PERROT T., PUSZTASZIERI M., PULETTI P. and BECKER M.: MRI with DWI for the detection of post-treatment head and neck squamous cell carcinoma: Why morphologic MRI criteria matter. AJNR Am. J. Neuroradiol., a5548: 1-8, 2018.

28- FURUKAWA M., PARVATHANENI U., MARAVILLA K., RICHARDS T. and ANZAI Y.: Dynamic contrastenhanced MR perfusion imaging of head and neck tumors at 3 Tesla. Head \& Neck, 35 (7): 923-9, 2013.

29- CHAWLA S., KIM S., LOEVNER L., HWANG W., WEINSTEIN G., CHALIAN A., et al.: Prediction of disease-free survival in patients with squamous cell carcinomas of the head and neck using dynamic contrastenhanced MR imaging. AJNR Am. J. Neuroradiol., 32: $778-84,2011$

30- AGRAWAL S., AWASTHI R., SINGH A. and HARIS M.: An exploratory study into the role of dynamic contrast- 
enhanced (DCE) MRI metrics as predictors of response in head and neck cancers. Clin. Radiol., 67: e1-5, 2012.

31- ISHIYAMA M., RICHARDS T., PARVATHANENI U. and ANZAI Y.: Dynamic contrast-enhanced magnetic resonance imaging in Head and Neck Cancer: Differentiation of new $\mathrm{H} \& \mathrm{~N}$ cancer, recurrent disease, and benign post-treatment changes. Clinical Imaging, 39 (4): 56670, 2015.

32- KING A., CHOW S., YU K., MO F., YEUNG D., YUAN J., et al.: DCE-MRI for Pre-Treatment Prediction and Post-Treatment Assessment of Treatment Response in Sites of Squamous Cell Carcinoma in the Head and Neck. PLOS ONE, 10 (12): e0144770, 2015.

\section{قيمة التصوير بالرنين المغناطيسى بإستخدام خاصيتى الإنتشاروالإرواء

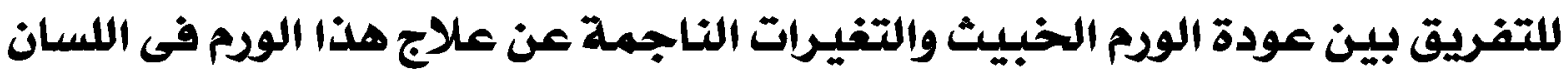

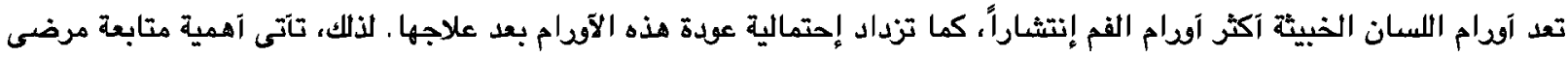

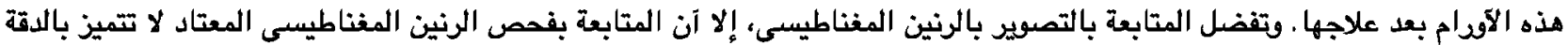

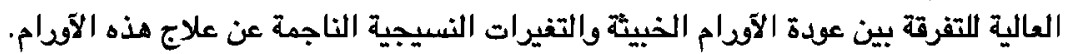

قد آثتبت عدة دراسات آن آشعة الرنين المغناطيسى بإستخدام خاصيتى الإنتشار والإرواء آسفرت عن نتائج مبشرة في التقرقة بين عودة

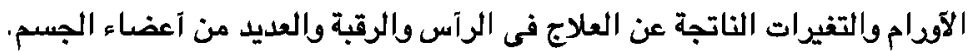

تهلف هذه الدراسة إلى تقييم لود آشعة الرنين المغناطيسي بإستخدام خاصيتى الإنتشار والإرواء بإستخدام الرنين المغناطيسى الديناميكى الرئي

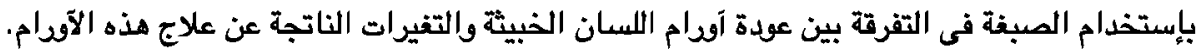

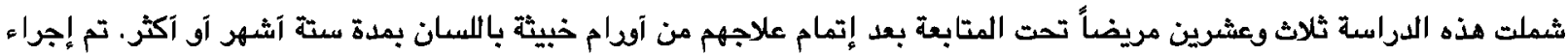

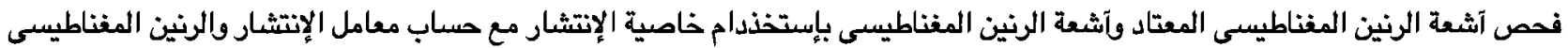

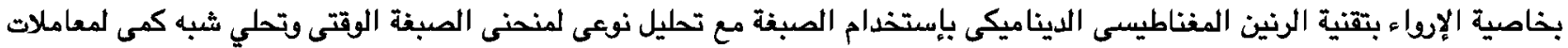

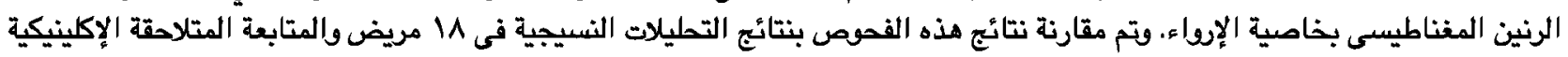
ويفحوص الآشعة فى ه مرضى كمرجعيات لهذه الدراسة.

وقد آثتبت هذه الدراسة آن فحص الرنين المغناطيسى بإستخدام خاصية الإنتثار وسيلة سريعة غير تداخلية تسفر عن نتائج دقية فى الري

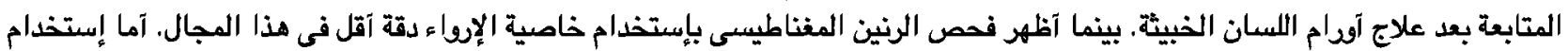
كلا الفحصين معاً آسفر عن نتائج أكثر دقة فى التفرتة بين عودة آورام اللسان الخييثة والتغيرات الناجمة عن علاج هذه الآورام. 